\title{
mGluR7 undergoes rapid internalization in response to activation by the allosteric agonist AMN082
}

\author{
Kenneth A. Pelkey ${ }^{\mathrm{a}, *, 1}$, Xiaoqing Yuan ${ }^{\mathrm{a}, 1}$, Gabriela Lavezzari ${ }^{\mathrm{b}}$, \\ Katherine W. Roche ${ }^{\mathrm{b}}$, Chris J. McBain ${ }^{\mathrm{a}}$ \\ ${ }^{a}$ Laboratory of Cellular and Synaptic Neurophysiology, National Institute of Child Health and Human Development, National Institutes of Health, \\ Building 35, 35 Lincoln Drive, Bethesda, MD 20892, USA \\ ${ }^{\mathrm{b}}$ National Institute of Neurological Disorders and Stroke, National Institutes of Health, Building 35, 35 Lincoln Drive, Bethesda, MD 20892, USA
}

Received 16 May 2006; received in revised form 10 July 2006; accepted 12 July 2006

\begin{abstract}
The G-protein coupled receptor (GPCR) metabotropic glutamate receptor 7 (mGluR7) is widely expressed throughout the nervous system and is implicated in diverse physiological processes ranging from synaptic plasticity to neuroprotection. To date, unequivocally assigning specific functions to mGluR7 has been hampered by a lack of specific pharmacological tools, however, an mGluR7 specific allosteric agonist, AMN082, was recently discovered. Accumulating evidence indicates that in addition to G-protein activation, GPCRs trigger critical intracellular signalling cascades during agonist-induced internalization. Thus, to determine if AMN082 will be useful for evaluating signalling events related to mGluR7 internalization as well as receptor activation we have examined whether AMN082 induces mGluR7 endocytosis. Using an immunofluorescence assay we demonstrate that AMN082 induces robust internalization of mGluR7 overexpressed in dissociated hippocampal neurons. AMN082-induced mGluR7 internalization was resistant to inhibition by a competitive antagonist consistent with the distinct binding site of the allosteric agonist from the glutamate-binding pocket utilized by conventional orthosteric ligands. Finally, as an independent assay of receptor internalization we overexpressed N-terminal pHluorin-tagged mGluR7 in neurons, allowing live imaging of surface receptors in real time. AMN082 treatment produced a rapid loss of surface mGluR7 as indicated by decreased fluorescence confirming the ability of allosteric receptor activation to trigger mGluR7 endocytosis. Thus, AMN082 will be effective for investigating physiological processes related to both mGluR7 activation and internalization such as control of bidirectional plasticity at mossy fiber-st. lucidum interneuron synapses.
\end{abstract}

(C) 2006 Elsevier Ltd. All rights reserved.

Keywords: mGluR7; Endocytosis; Internalization; Allosteric; GPCR; pHluorin

\section{Introduction}

Glutamate is the major excitatory neurotransmitter throughout the central nervous system (CNS) acting on both ionotropic (iGluRs) and metabotropic glutamate receptors (mGluRs). Whereas iGluRs primarily mediate fast moment to moment synaptic communication by allowing postsynaptic ion flux upon activation, mGluR signaling generates slower,

\footnotetext{
* Corresponding author. Tel.: +1 301402 1985; fax: +1 3014024777

E-mail address: pelkeyk2@mail.nih.gov (K.A. Pelkey).

${ }^{1}$ These authors contributed equally to the work.
}

longer lasting changes in intracellular signaling cascades by activating associated $\mathrm{G}$ proteins upon glutamate binding. Importantly, ionotropic and metabotropic components of transmission are intimately related as mGluR signaling frequently modulates the efficacy of iGluR-mediated transmission. One mechanism by which mGluRs regulate fast transmission is to depress glutamate release, a function typically performed by presynaptically expressed mGluR autoreceptors (reviewed in Conn, 2003; Schoepp, 2001). This mGluR autoreceptormediated presynaptic depression yields a powerful negative feedback loop that may prevent runaway excitation. Additionally, presynaptic mGluRs serve as critical molecular switches for induction of presynaptically expressed short- and long-term 
synaptic plasticity (e.g. Alexander and Godwin, 2005; Billups et al., 2005; Pelkey et al., 2005; Tzounopoulos et al., 1998). Thus, given the widespread utilization of mGluRs at glutamatergic terminals throughout the CNS, it is of great interest to elucidate cellular mechanisms controlling presynaptic mGluR function.

mGluRs belong to the GPCR superfamily and comprise eight receptor subtypes (mGluR1-8) classified into three major groups (reviewed in Conn and Pin, 1997; Pin and Acher, 2002). Group I mGluRs consisting of mGluR 1 and 5 are primarily expressed postsynaptically and couple to phosphoinositide hydrolysis through $\mathrm{G}_{\mathrm{q}}$ activation. In contrast group II mGluRs consisting of mGluR2 and 3 along with group III mGluRs consisting of mGluRs4,6,7, and 8 generally subserve presynaptic autoreceptor function and couple to adenylyl cyclase (AC) inhibition through $G_{i}$ activation. One ubiquitous regulatory mechanism controlling GPCR function is agonistinduced desensitization, a complex process typically involving receptor phosphorylation, $\mathrm{G}$ protein-uncoupling, and ultimately receptor internalization (reviewed in Claing et al., 2002; Ferguson, 2001). Surprisingly, despite a wealth of information concerning detailed molecular mechanisms of desensitization/internalization for many GPCRs such as adrenergic receptors, research into mGluR desensitization/internalization, particularly for group II and III autoreceptor mGluRs, has noticeably lagged.

Recently we reported that mGluR7 localized to hippocampal mossy fiber (MF) terminals innervating stratum lucidum interneurons (SLINs) functions as a metaplastic switch whose surface expression governs bidirectional MF-SLIN plasticity (Pelkey et al., 2005). At naïve MF-SLIN synapses surface mGluR7 activation following high-frequency stimulation (HFS) generates long-term depression (LTD) by irreversibly reducing presynaptic release. However, agonist exposure causes mGluR7 internalization, which unmasks the ability of MFs to persistently enhance release at SLIN inputs after HFS yielding presynaptic long-term potentiation (LTP). Thus, regulated mGluR7 trafficking critically determines the polarity of MF-SLIN plasticity.

Investigation of mGluR7 function has been greatly hampered by the lack of specific pharmacological tools (Conn and Niswender, 2006). The group III mGluR agonist L-AP4 is frequently utilized to ascribe a particular function to this family of receptors. However, L-AP4 activates mGluR7 only at concentrations $2-3$ orders of magnitude higher than those able to activate mGluRs 4,6 , and 8 making it impossible to study the physiological effects of mGluR7 without potentially confounding effects induced by the higher affinity related mGluRs (Conn and Pin, 1997). A recent report characterized the first ever mGluR7 specific agonist AMN082 (Conn and Niswender, 2006; Mitsukawa et al., 2005). Interestingly, AMN082 acts via a unique mechanism fully activating mGluR7 through an allosteric site removed from the glutamate binding pocket (Mitsukawa et al., 2005). The effects of allosteric reagents, including those with full receptor activating properties, on receptor internalization can differ greatly from conventional orthosteric ligands (Klaasse et al., 2005; May et al., 2005; Sachpatzidis et al., 2003; Toulme et al., 2006). Thus, it is not immediately apparent whether AMN082 will be useful in evaluating physiological events related to mGluR7 endocytosis such as the conversion of depressing to potentiating MF-SLIN synapses. In the present investigation we have examined the ability of AMN082 to induce internalization of mGluR7 overexpressed in dissociated hippocampal neurons.

\section{Materials and methods}

\subsection{Fluorescence-based antibody uptake internalization assay}

Methods for the assay are described in detail elsewhere (Lavezzari et al., 2004). Briefly, cultured hippocampal neurons (10 DIV) were transfected with $\mathrm{N}$-terminally myc-tagged mGluR7a (Perroy et al., 2000) using the calcium phosphate coprecipitation method. After $48 \mathrm{~h}$, cultures were washed in PBS and incubated with monoclonal anti-myc antibody (9E10, Santa Cruz Biotechnology) for $45^{\prime}$ at room temperature to label surface-expressed receptors. Following removal of the anti-myc antibody, cultures were returned to conditioned media supplemented with or without indicated reagents for $15^{\prime}$ at $37^{\circ} \mathrm{C}$. Cells were washed in PBS and fixed (4\% paraformaldehyde $/ 4 \%$ sucrose in PBS for $15^{\prime}$ ) then incubated for $30^{\prime}$ at room temperature with Alexa 568-conjugated (red) anti-mouse secondary antibody (Molecular Probes, Eugene, Oregon) to label the surface population. After removal and wash of the red secondary antibody, cells were permeabilized $(0.25 \%$ Triton-X-100 in PBS for $5 \mathrm{~min}$ ), incubated with blocking solution (10\% normal goat serum for $30^{\prime}$ ), then incubated with Alexa 488-conjugated (green) anti-mouse secondary antibody $\left(30^{\prime}\right)$ to label myc-mGluR7 that was internalized with bound primary. After washing in PBS cells were mounted (ProLong Antifade Kit, Molecular Probes) and imaged with a $63 \times$ objective on a Zeiss LSM 510 confocal microscope. Alexa 568 dye was excited with a $543 \mathrm{~nm}$ $\mathrm{HeNe}$ laser and the emission was collected beyond $560 \mathrm{~nm}$. Alexa 488 dye was excited with an Ar $488 \mathrm{~nm}$ laser and the emission was collected between 500-550 nm. No cross talk between channels was detected with these settings. Serial optical sections collected at $0.34 \mu \mathrm{m}$ intervals were used to create maximum projection images that were used for quantitative analysis with Volocity 2 software (Improvision). Quantified internalization (green voxels not colocalized with red) was assessed in at least three independent experiments (from three independent hippocampal preparations) for each condition. In a given experiment $3-4$ processes $(10-15 \mu \mathrm{m})$ were randomly selected from 3-4 transfected neurons for each condition (9-16 processes per condition per experiment). Data are presented as percentage of control internalization assessed in cultures incubated with non-supplemented conditioning media. Statistical significance was assessed using $t$-tests, with similar results $(p<0.01)$ obtained by counting each process as an independent observation or restricting the degrees of freedom by using a mean of means for each experimental condition.

\section{2. pHluorin-mGluR7 based internalization assay}

\subsubsection{Construction of pHluorin-mGluR7 DNA vectors and Sindbis viruses} The 720bp coding sequence of superecliptic pHluorin was excised from the pCR4TOPO-pHluorin construct (a gift from Dr. Jeremy Henley's lab) using AscI digestion. The myc-tag sequences of the mGluR7a and mGluR7b constructs (provided by Dr. L Fagni; Perroy et al., 2000) were removed and an $A s c$ I site was engineered between the $60 \mathrm{bp}$ sequence encoding the signal peptide of mGluR5 and the cDNA of mGluR7a/b by PCR-based site-directed mutagenesis (ExSite PCR-Based Site-Directed Mutagenesis Kit, Stratagene, La Jolla, CA). Then the pHluorin fragment was inserted at the AscI site to generate constructs pRK5-pHluorin-mGluR7a/b, which express as mGluR7a/b with $\mathrm{N}$-terminal associated pHluorin. Subsequently pHluorin-mGluR7a/b fragments were excised using $E c o \mathrm{R} 1$, blunt ended, and inserted into the PmlI site of modified Sindbis vector SINrep (nsP2S726; Kim et al., 2004). All restriction enzymes were from New England Biolabs, Inc. Beverly, MA. 


\subsubsection{Hippocampal primary cultures}

Hippocampal primary neurons were prepared from E18 rats using standard methodology. Briefly, dissociated hippocampal cells were plated on poly-Llysine coated coverslips at a density of 200 cells $/ \mathrm{mm}^{2}$ (typically, 200,000 cells per $25 \mathrm{~mm}$ coverslip in 1 well of 6-well plate) in Neurobasal medium supplemented with B27 (NBMC; all from Gibco-BRL), and maintained under $37^{\circ} \mathrm{C}$, $5 \% \mathrm{CO}_{2}$. Cells were cultured for 2 weeks before infection with $10 \mu \mathrm{M} \mathrm{AraC}$ being added at 5 DIV to eliminate glia.

\subsubsection{Sindbis virus infections}

RNA of SINrep-pHluorin-mGlurR7a/b and the optimized helper vector, DH-BB (Kim et al., 2004), were electroporated into BHK cells. Pseudovirion-containing medium was collected after $24 \mathrm{~h}$ of transfection. Viruses were concentrated and purified from the BHK culture medium using micron100 (Millipore).

Primary cultured hippocampal neurons on $25 \mathrm{~mm}$ coverslips were infected by $0.5-1 \mu \mathrm{l}$ virus stock in $700 \mu \mathrm{l}$ NBMC for $1 \mathrm{~h}$. Cultures were then washed and incubated in NBMC and allowed to recover for $12-48 \mathrm{~h}$ until imaging. Toxicity of infected neurons, assessed by visual inspection as well as acid and $\mathrm{NH}_{4} \mathrm{Cl}$ washes, was minimal within this time frame.

\subsubsection{Live cell imaging}

Infected hippocampal neurons were imaged using an LSM510 META confocal system (Zeiss, Germany) in the NICHD microscopy and imaging core (MIC) facility. Cells were transferred to HEPES-buffered saline (HBS) containing (in $\mathrm{mM}$ ): $25 \mathrm{HEPES}, 130 \mathrm{NaCl}, 10$ glucose, $5.3 \mathrm{KCl}, 1.5 \mathrm{MgCl}_{2}$, and $2.0 \mathrm{CaCl}_{2}, \mathrm{pH} 7$.4. Indicated reagents from frozen stocks were added directly to $\mathrm{HBS}$ aliquots and osmolarity and $\mathrm{pH}$ were readjusted as required. For treatment with $\mathrm{NH}_{4} \mathrm{Cl}(50 \mathrm{mM})$ an equimolar amount of $\mathrm{NaCl}$ was removed from HBS. Reagents were applied by hand-fed exchange of non-supplemented HBS bathing cultures for HBS containing indicated drugs. Imaging was performed at room temperature using a Neofluar $40 \times / 1.3 \mathrm{NA}$ oil or a PlanApochromat $63 \times / 1$.4NA oil objective mounted on an inverted Zeiss Axiovert $200 \mathrm{M}$ microscope. pHluorin-mGluR7a/b was excited by a $488 \mathrm{~nm}$ laser, and emission was detected through a 505 long-pass filter. Z-stacks with depth between $0.3 \mu \mathrm{m}-0.5 \mu \mathrm{m}$ were collected at regular intervals $(1-10 \mathrm{~min})$ during the course of a $30-50 \mathrm{~min}$ imaging session. For analysis stacks for each time point of a given experiment were compressed as maximum projection images and fluorescence intensity in randomly chosen regions of interest (4-10 ROIs per experiment) across neuronal processes was measured using MetaMorph image analysis software (Universal Imaging Corporation, West Chester, PA). For each data set experiments were repeated in a minimum of 5 different cultures, thus yielding a minimum of 20 observations per condition ( 5 experiments times 4 ROIs). Quantified results are expressed as percentage of control fluorescence intensity obtained at the beginning of each imaging session, allowing statistical significance to be determined via paired t-tests. The majority of experiments were performed using pHluorin-mGluR7b. However, as similar results were observed in a limited number of experiments with pHluorin-mGluR7a the data have been pooled which we refer to as a results for pHluorin-mGluR7.

\subsubsection{Immunocytochemistry}

Cells infected with pHluorin-mGluR7 were fixed with $4 \%$ paraformaldehyde at room temperature for $10 \mathrm{~min}$. Surface pHluorin-mGluR7 was labeled with mouse anti-GFP antibody (Chemicon, Temecula, CA), incubated at room temperature for $1 \mathrm{~h}$ in PBS supplemented with $1 \%$ goat serum. Following wash in PBS the primary labeled cultures were incubated with Alexa-555 conjugated secondary (Molecular Probes. Eugene, OR) at room temperature for $30 \mathrm{~min}$. Subsequently, cells were thoroughly washed again (PBS), treated once more with $4 \%$ paraformaldehyde at room temperature for $10 \mathrm{~min}$ then permeablized with $0.1 \%$ Triton $100-\mathrm{X}$ at room temperature for $10 \mathrm{~min}$ to ensure similar $\mathrm{pH}$ (7.4) in all cellular compartments. Coverslips were mounted with Mowiol, and imaged using an LSM510 META confocal system through a Neofluar $40 \times / 1.3 \mathrm{NA}$ oil objective. pHluorin was excited with a $488 \mathrm{~nm}$ argon ion laser and emission collected through a $505-530 \mathrm{~nm}$ bandpass filter. Alexa-555 was excited by a $543 \mathrm{~nm}$ Helium-Neon laser, $1 \mathrm{~mW}$ and emission was collected through a $560-615 \mathrm{~nm}$ bandpass filter. Images are shown as maximum projections.

\section{Results}

\section{1. mGluR7 internalization revealed by immunofluorescence}

To initially examine agonist-induced internalization of mGluR7 we utilized a fluorescence-based antibody uptake internalization assay in dissociated hippocampal cultured neurons transfected with $\mathrm{N}$-terminally myc-tagged mGluR7a (Lavezzari et al., 2004; Pelkey et al., 2005). Cultures were incubated with monoclonal anti-myc antibody to label mycmGluR7 expressed on the surface of transfected neurons. Following removal of the anti-myc primary antibody cultures were returned for $15 \mathrm{~min}$ to conditioned media supplemented with or without various reagents as indicated allowing for endocytosis of surface myc-mGluR7 with associated primary antibody. Subsequently, cultures were fixed and incubated with Alexa-568 conjugated secondary antibody to label antimyc associated receptors that remained on the surface with a red fluorophore. Removal of the red secondary antibody, followed by membrane permeabilization and incubation with Alexa-488 conjugated secondary antibody then allowed specific labeling of internalized receptors with a green fluorophore. Using this methodology a small percentage of surface mGluR7 can be observed to constitutively internalize following incubation in non-supplemented medium which we designate the control baseline level of receptor endocytosis (Figs. 1A, B). Consistent with our previous observations (Pelkey et al., 2005) supplementing the conditioning medium with the group III mGluR orthosteric agonist L-AP4 $(400 \mu \mathrm{M})$ dramatically increased receptor endocytosis as indicated by both a loss of remaining surface stain (red) and an increase in the intracellular stain (green; Fig. 1A). Also as previously reported (Pelkey et al., 2005) the L-AP4-induced increase in mGluR7 internalization was prevented when cultures were concomitantly treated with the competitive group III mGluR antagonist MSOP (100-400 $\mu \mathrm{M}$, Fig. 1A), indicating that the L-AP4 effect is specific to receptor binding and activation.

Having confirmed our previous observations we next examined whether the newly characterized mGluR7-specific allosteric agonist AMN082 causes receptor endocytosis. AMN082 $(0.5-1 \mu \mathrm{M})$ treatment produced strikingly robust mGluR7 internalization: supplementing media with AMN082 increased receptor internalization to $291 \pm 30 \%$ of control levels observed in transfected sister cultures incubated with non-supplemented media that were run in parallel (Figs. 1B, C). As the mGluR7 AMN082 binding site is distinct from the glutamate binding pocket of the receptor, conventional competitive antagonists do not prevent receptor activation by the allosteric agonist (Mitsukawa et al., 2005). Similarly, MSOP failed to prevent AMN082-induced mGluR7 endocytosis: receptor internalization was $205 \pm 28 \%$ of control internalization assessed in parallel experiments using vehicle treated transfected sister cultures (Figs. 1B, C). At present there are no known antagonists of AMN082 to test. These findings reveal that the allosteric agonist AMN082 does indeed induce mGluR7 internalization indicating that this reagent may be useful for characterizing not only 
A
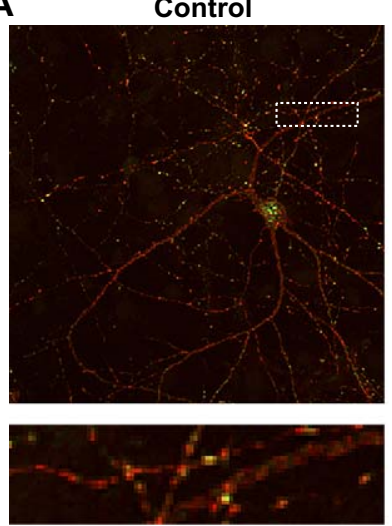

B
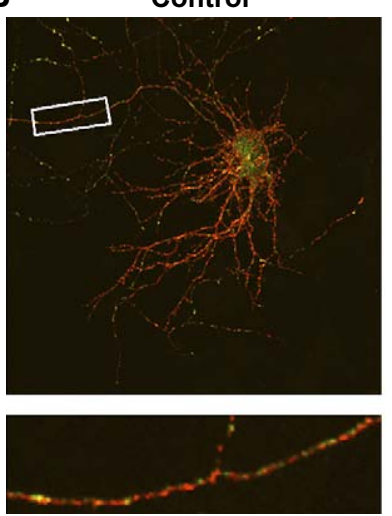

C
L-AP4
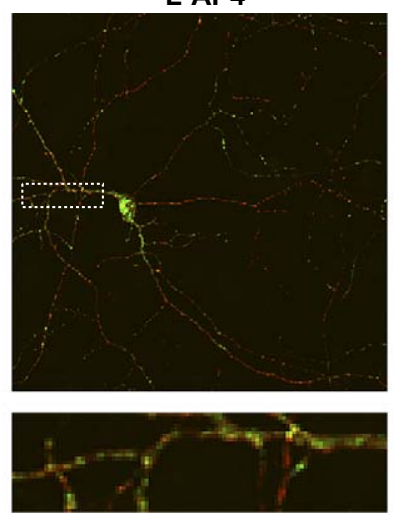

AMN082
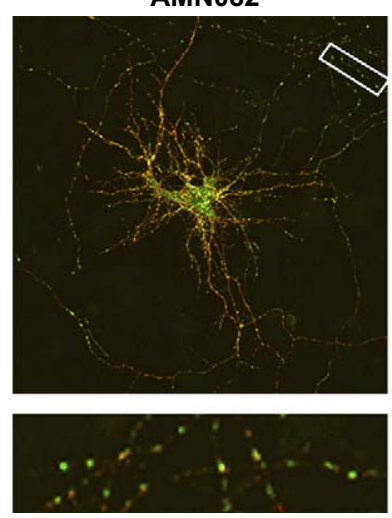

**

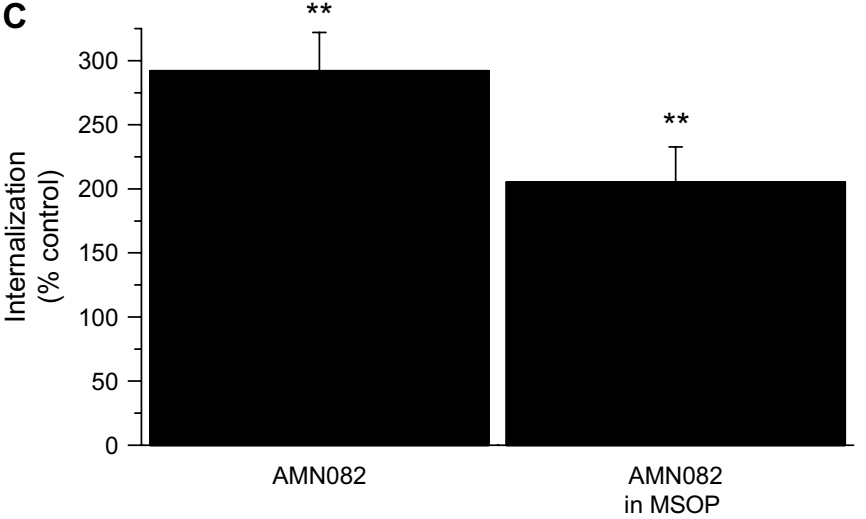

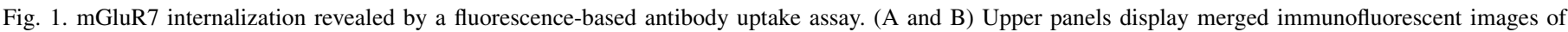

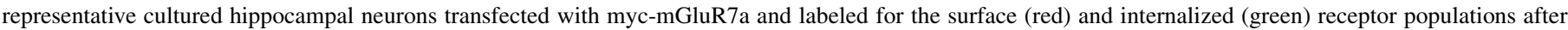

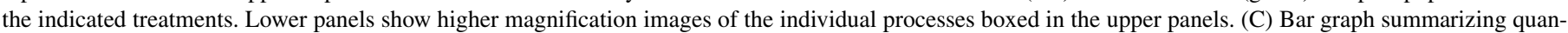

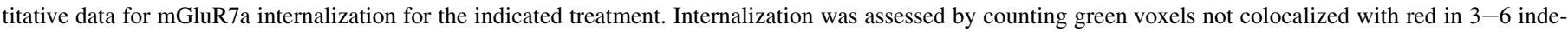

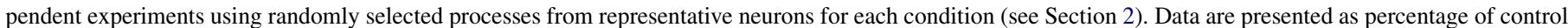

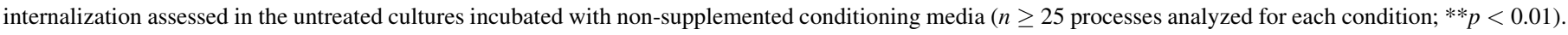

mGluR7 activation related cell signaling events (Mitsukawa et al., 2005) but also signals generated as a consequence of receptor endocytosis (Ferguson, 2001; Lefkowitz and Shenoy, 2005; Pelkey et al., 2005).

\subsection{Real time imaging of ecliptic pHluorin tagged $m$ GluR7 internalization}

Our previous electrophysiological studies suggest that the duration of mGluR7 internalization following agonist exposure may critically regulate the temporal window for evoking MF-SLIN LTP as receptor reinsertion into presynaptic membranes should prevent LTP induction and promote further depression in response to HFS (Pelkey et al., 2005). In these studies we could reliably induce LTP when HFS was administered up to $10 \mathrm{~min}$ following agonist removal. Furthermore, MF-SLIN transmission remained desensitized to a second application of L-AP4 for a similar period of time (Pelkey et al., 2005). Thus, if mGluR7 internalization is indeed a key requirement for converting depressing MF-SLIN synapses into 
potentiating ones, the receptors should not rapidly return to the membrane following agonist-induced internalization as has been reported for mGluR4 (Iacovelli et al., 2004).

To examine the cell surface dynamics of mGluR7 in real time before, during, and after agonist treatment we infected cultured hippocampal neurons with a viral construct encoding mGluR7 N-terminally tagged with a $\mathrm{pH}$-sensitive GFP (superecliptic pHluorin; Ashby et al., 2004; Bouschet et al., 2005; Jacob et al., 2005; Kopec et al., 2006; Miesenbock et al., 1998; Yudowski et al., 2006). Recombinant, GFP-tagged receptors have been widely used to investigate the trafficking dynamics of a number of GPCRs. However, this approach is limited, particularly in fine neuronal processes, by an inability to directly distinguish surface from intracellular fluorescence. In contrast $\mathrm{N}$-terminally expressed pHluorin principally reveals only the surface population of tagged receptors as fluorescence associated with the intracellular population is quenched in the acidic lumen of vesicular compartments (Miesenbock et al., 1998; Sankaranarayanan and Ryan, 2000). Furthermore, the rapid acidification of endocytic vesicles upon membrane fission (Demaurex, 2002) allows receptor internalization to be accurately reported as a decrease in fluorescence intensity (Ashby et al., 2004; Yudowski et al., 2006).

The vast majority of our experiments were performed with pHluorin-mGluR7b to complement our findings from the immunofluorescence assay performed with mGluR7a. However, similar results were observed with pHluorin-mGluR7a in a limited number of experiments and, thus, the data have been pooled and are referred to as results for pHluorin-mGluR7. Immunostaining of fixed cultures with anti-GFP antibody under non-permeabilizing conditions revealed robust pHluorinmGluR7 surface expression throughout infected neurons beyond $12 \mathrm{~h}$ after viral infection with minimal toxicity up to $50 \mathrm{~h}$ post-infection (Figs. 2A, B). However, as numerous intracellular compartments exhibiting varying degrees of acidification exist near the somatic region of neurons we focused our live imaging experiments on neuronal processes in which intracellular receptors principally reside on acidified secretory and endocytic vesicles. When processes of infected neurons were imaged at regular intervals $(2-10 \mathrm{~min})$ with confocal microscopy fluorescence intensity remained stable for up to $1 \mathrm{~h}$ in cultures bathed in standard Hepes buffered saline (HBS, $\mathrm{pH}$ 7.4) indicating that minimal photobleaching occurs during the course of an experiment: after $40 \mathrm{~min}$ of imaging fluorescence intensity was $115 \pm 3.8 \%$ of control fluorescence intensity measured within the first minute of the recording (data not shown). The inability to observe constitutive pHluorinmGluR7 internalization as a loss of fluorescence likely reflects an equilibrium between receptor endocytosis and insertion in the absence of any stimulation. Fluorescence intensity in processes of infected neurons was reversibly depressed to $52 \pm 2.9 \%$ of control during brief acidification ( $\mathrm{pH}$ 6.0) of the extracellular environment (Figs. 2C, E). In contrast, treatment with $\mathrm{NH}_{4} \mathrm{Cl}$ to collapse intracellular $\mathrm{pH}$ gradients significantly increased fluorescence intensity to $148 \pm 7.0 \%$ of control in neutral HBS and this increased signal was sensitive to concomitant acid treatment (Figs. 2D, E). Together these control experiments indicate that in neutral HBS visible fluorescence accurately reports surface pHluorin-mGluR7 with signals from the intracellular population of receptors being effectively quenched in acidic vesicular compartments.

Consistent with the data obtained from our fluorescencebased antibody uptake assays, treatment of infected neurons with L-AP4 $(400 \mu \mathrm{M})$ caused significant internalization of pHluorin-mGluR7 as indicated by a dramatic reduction in fluorescence intensity (Fig. 3). Internalization could frequently be observed within the first 2 min of agonist treatment and typically reached maximal levels within 5-10 $\mathrm{min}$ of exposure (Figs. 3A, B). Quantitation within multiple regions of interest across processes of individual neurons from different cultures indicated that fluorescence intensity decreased to $47.5 \pm 2.3 \%$ of control levels within $10 \mathrm{~min}$ of agonist treatment (Fig. 3C). The L-AP4-induced loss of fluorescence was prevented by MSOP $(115 \pm 3.9 \%$ of control after 5-10 min of concomitant L-AP4 and MSOP) indicating that the effect of L-AP4 is specific to receptor binding and activation (data not shown). Importantly, there was no significant recovery of fluorescence within $10 \mathrm{~min}$ of agonist removal (fluorescence intensity remained at $47.1 \pm 2.0 \%$ of control; Figs. $3 \mathrm{~A}-\mathrm{C}$ ), consistent with a lack of rapid pHluorin-mGluR7 reinsertion during the time frame that we have observed to be permissible for LTP generation at MF-SLIN synapses (Pelkey et al., 2005). The lack of fluorescence recovery after agonist removal did not result from cell death or photobleaching as the internalized receptors, along with the initially quenched intracellular population, could be revealed with $\mathrm{NH}_{4} \mathrm{Cl}$ treatment at the end of each experiment (Figs. 3A-C).

In a final series of experiments we reexamined the ability of the allosteric mGluR7 agonist AMN082 to cause receptor internalization using the pHluorin-mGluR7 based assay (Fig. 4). Similar to our observations with L-AP4, AMN082 (0.5$1 \mu \mathrm{M})$ treatment produced a robust decrease in the fluorescence intensity of pHluorin-mGluR7 expressing neuronal processes that was typically evident within $5 \mathrm{~min}$ of agonist application (58.0 $\pm 3.0 \%$ of control; Figs. 4A-D). Once again there was no significant fluorescence recovery within $10 \mathrm{~min}$ following agonist removal and subsequent $\mathrm{NH}_{4} \mathrm{Cl}$ application increased fluorescence intensity beyond the initial control levels (Figs. 4A-D). Considered together these findings confirm the effectiveness of AMN082 to cause mGluR7 internalization suggesting that receptor activation rather than conformational changes associated with glutamate binding drive efficient receptor internalization.

\section{Discussion}

We have examined whether the newly discovered mGluR7 specific agonist AMN082 promotes receptor internalization. Using two independent assays we found that receptor activation via this allosteric agonist does indeed cause mGluR7 internalization. Thus, AMN082 will be an effective tool for investigating physiological processes related to both mGluR7 activation (i.e. conventional G-protein coupling) as well as signaling cascades triggered by mGluR7 internalization such as 
A

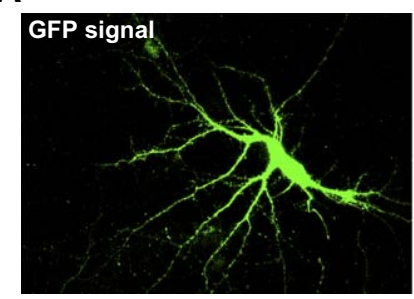

B

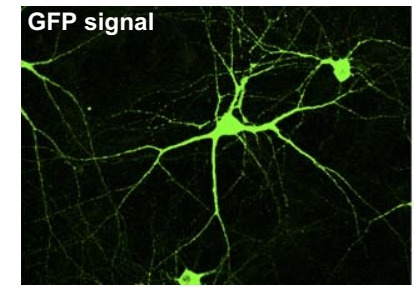

C

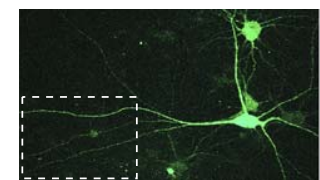

D

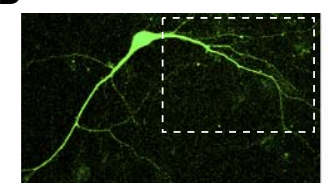

12 hours post-infection

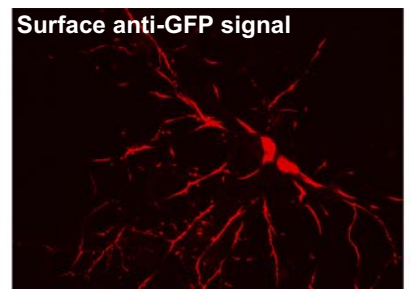

24 hours post-infection

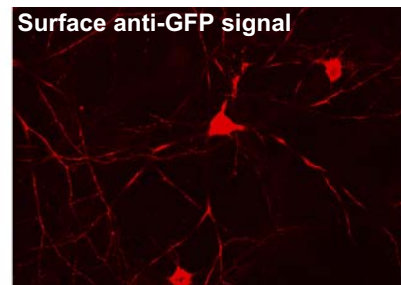

Control pH 7.4

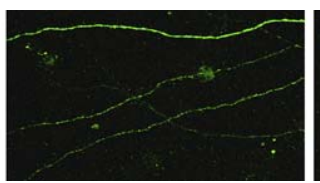

Control pH 7.4
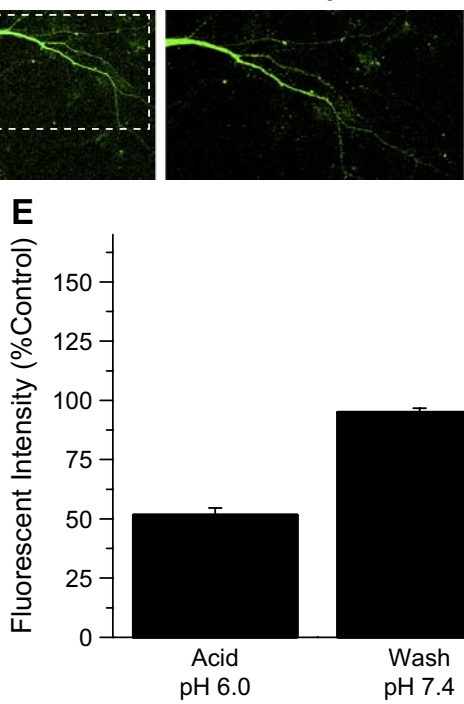

Acid pH 6.0

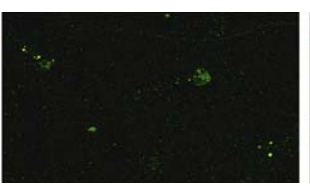

$\mathrm{NH}_{4} \mathrm{Cl}$ pH 7.4
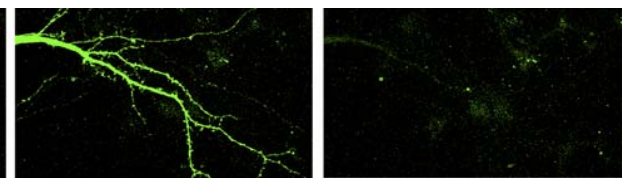

Wash pH 7.4

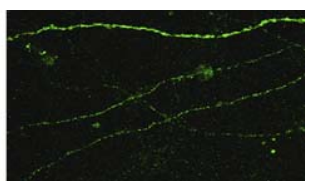

$\mathrm{NH}_{4} \mathrm{Cl}$ pH 6.0
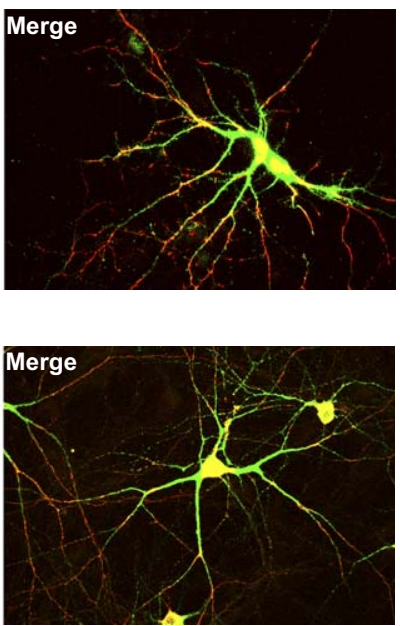

\section{(n)}




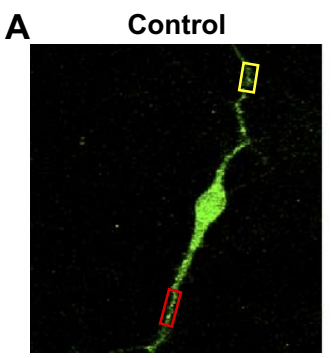

B

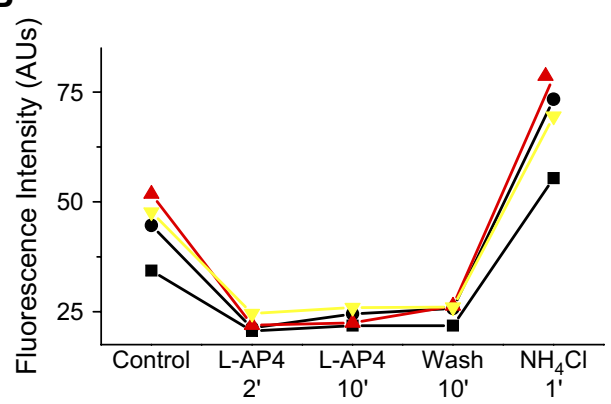

L-AP4 10'

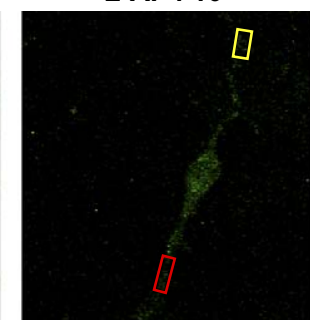

C

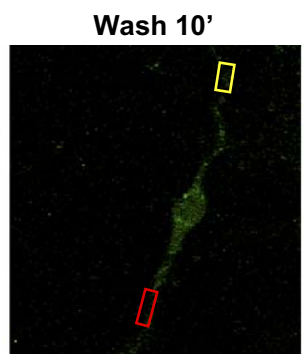

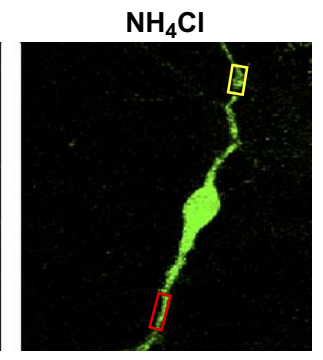

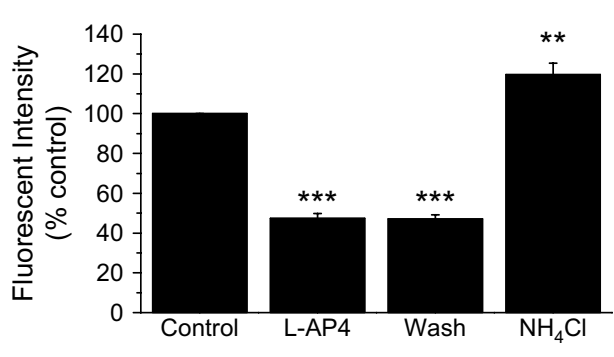

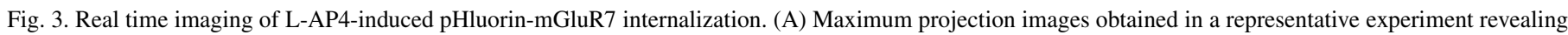

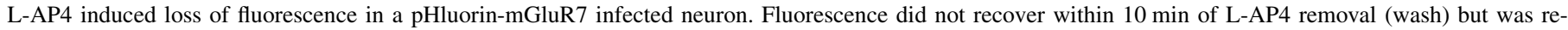

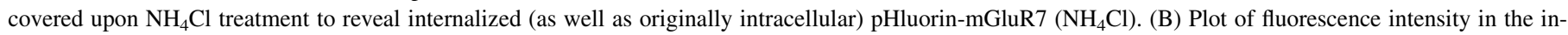

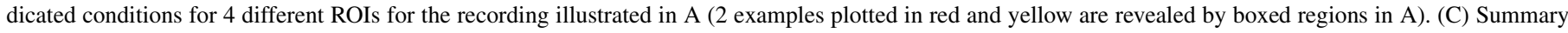

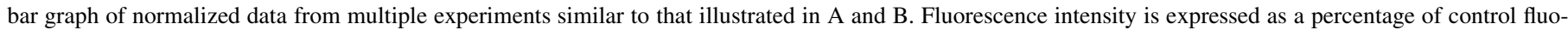

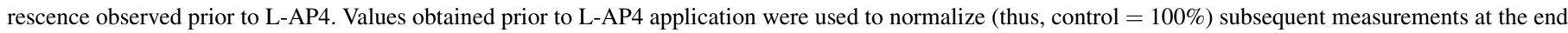

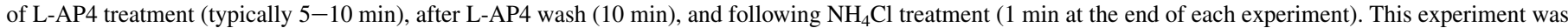
repeated in 6 different cultures and a total of 30 ROIs were examined. $* * * p<0.001$; **p $p 0.01$ compared to control.

agonist discovered, and it is orally active readily penetrating the blood-brain barrier (Mitsukawa et al., 2005). Our results, however, indicate that AMN082 will cause mGluR7 desensitization. This likely relates to the ability of AMN082 to fully activate mGluR7 in contrast to most allosteric modulators which do not directly activate receptors but rather enhance activation by orthosteric agonists or act as non-competitive antagonists. In contrast to our findings, activation of CXCR4 chemokine receptors by an allosteric agonist does not cause receptor internalization (Sachpatzidis et al., 2003), indicating that GPCRs are not always internalized by allosteric agonists.

Aside from our previous report of mGluR7 internalization (Pelkey et al., 2005), we know of only a handful of studies that have directly examined agonist-induced group III mGluR internalization. Prolonged agonist exposure (60 min) was reported to cause significant internalization of endogenous mGluR8 expressed in enteric neurons which could be prevented by co-application of a competitive group III mGluR antagonist (Tong and Kirchgessner, 2003). As shorter treatment periods were not examined, it is difficult to draw comparison with the current findings of rapid agonist-induced mGluR7 internalization; however, the report is consistent with the ability to observe agonist-induced internalization of group III mGluRs expressed on neuronal surfaces. Overexpressed GFP-tagged mGluR4 was found to undergo agonist-induced redistribution in HEK cells in a fashion consistent with receptor internalization (Iacovelli et al., 2004). Similar to our results, GFP-mGluR4 internalization occurred within $5 \mathrm{~min}$ of agonist treatment, however, fluorescence returned to the surface membrane within 10 min of continued agonist exposure (Iacovelli et al., 2004). As the ability of mGluR7 to remain internalized following agonist treatment is critical to our working model of bidirectional plasticity at MF-SLIN synapses we developed the pHluorin-mGluR7 constructs to specifically assay whether mGluR7 rapidly returns to neuronal surface membranes after agonist-induced internalization. Following internalization by either L-AP4 or AMN082 we did not observe fluorescence recovery in the continued presence of agonist, nor following agonist removal. This discrepancy between the findings with mGluR4 and mGluR7 may reflect inherent differences between the receptors themselves or perhaps differences between trafficking in neurons and a heterologous expression system.

A recent study using immunocytochemical techniques failed to observe agonist-induced internalization of mGluR4 expressed in HEK cells where group III mGluRs preferentially couple to $A C$ inhibition through $\mathrm{G}_{\mathrm{i}}$ (Mathiesen and Ramirez, 2006). Rather, mGluR4 underwent heterologous internalization following manipulations that activated PKC such as phorbol ester treatment or activation of a co-expressed $\mathrm{G}_{\mathrm{q}}$-coupled GPCR (NK3R; Mathiesen and Ramirez, 2006). Such heterologous internalization may explain the ability of PKC activation to reduce group III mGluR-mediated inhibition of $\mathrm{Ca}^{2+}$ currents and synaptic transmission (Macek et al., 1998; Swartz et al., 1993). Mathiesen and Ramirez (2006) also demonstrated significant agonist induced internalization when mGluR4 coupling was redirected from $\mathrm{AC}$ to $\mathrm{PLC} / \mathrm{PKC}$ signaling in HEK cells by co-expression of a chimeric $G_{\alpha q 05}$ protein. 
A

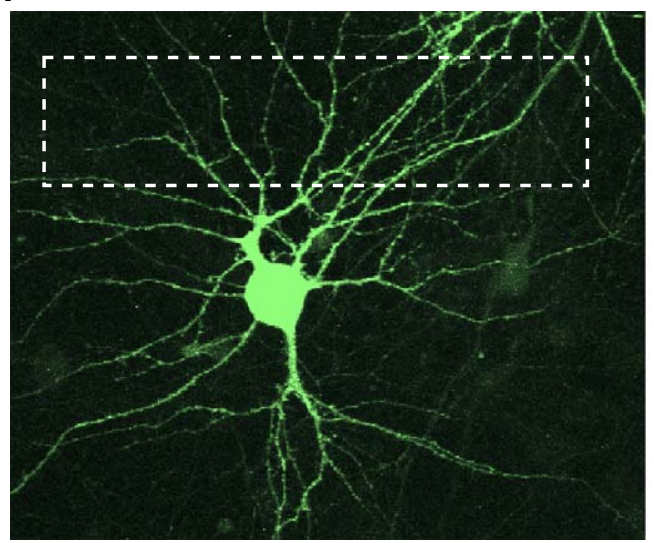

C

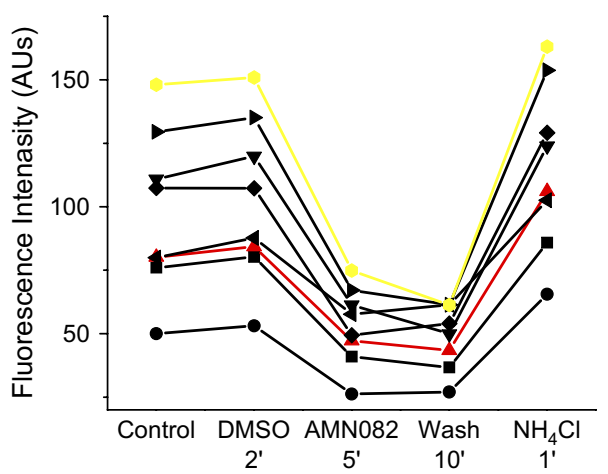

B

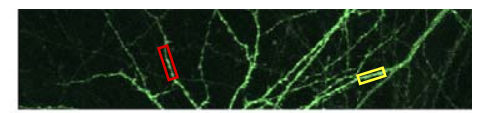

AMN082 5'

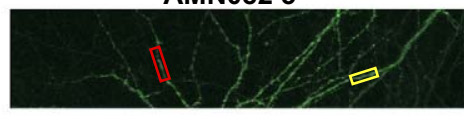

Wash 10'

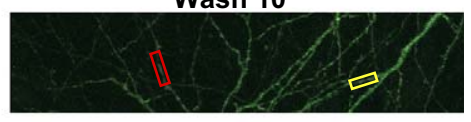

$\mathrm{NH}_{4} \mathrm{Cl}$

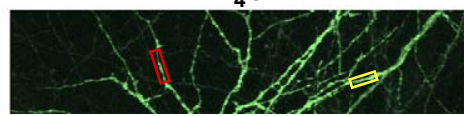

D

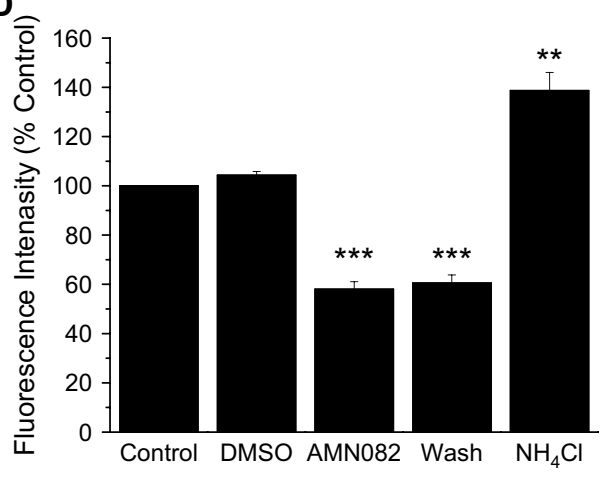

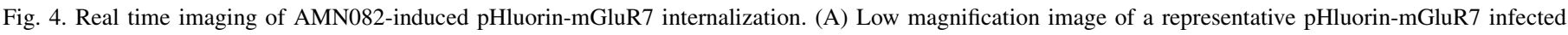

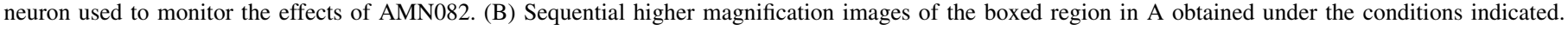

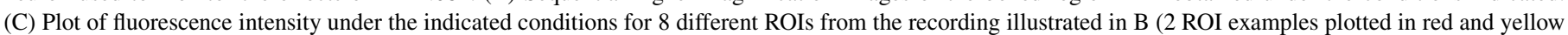

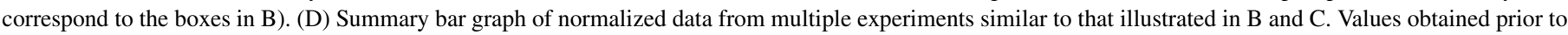

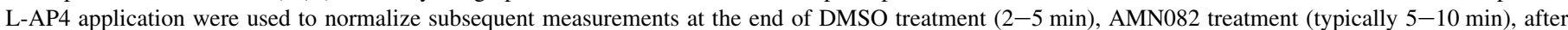

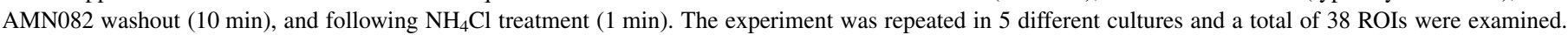
$* * * p<0.001 ; * * p<0.01$ compared to control.

Interestingly, while group III mGluRs readily couple to inhibition of AC through $G_{i}$ in heterologous expression systems there remains some question as to whether this is the preferred coupling mode of native receptors (Conn and Pin, 1997). Of particular relevance, mGluR7-mediated inhibition of MFSLIN synapses is prevented by PKC inhibition and unaffected by PKA inhibition (Pelkey et al., 2005). Furthermore, mGluR7-mediated depression of P/Q-type $\mathrm{Ca}^{2+}$ channels in cerebellar granule cells proceeds via a $\mathrm{G}_{\mathrm{o}} / \mathrm{PLC} / \mathrm{IP}_{3} / \mathrm{PKC}$ pathway (Perroy et al., 2000). These findings suggest that in a native environment mGluR7 (and perhaps higher affinity group III mGluRs) efficiently couples to a PLC/IP3/PKC pathway through $G_{o}$ rather than inhibition of $A C$ through $G_{i}$ (see also Iacovelli et al., 2002; Schoppa and Westbrook, 1997; Shen and Slaughter, 1998). Thus, the robust agonist-induced mGluR7 internalization observed in neurons here may reflect efficient PKC coupling while the failure to observe agonistinduced mGluR4 internalization in HEK cells (Mathiesen and Ramirez, 2006) would reflect inefficient PKC coupling. Indeed the relative abundance of $\mathrm{G}_{\mathrm{o}}$ compared to other $\mathrm{G}$ proteins in the CNS might dictate this difference between neurons and heterologous expression systems (Sternweis and
Robishaw, 1984). Additionally, numerous protein interactions of the intracellular C-terminal tail of mGluR7 (reviewed in Dev et al., 2001) that are not replicated in heterologous expression systems are likely to influence regulated receptor trafficking in neurons.

In contrast to the sparsity of information concerning internalization of typical presynaptic autoreceptor mGluRs (groups II and III), reports investigating internalization of conventionally postsynaptic group I mGluRs has accumulated in recent years (reviewed in Dhami and Ferguson, 2006). Similar to our findings both mGluR1 and mGluR5 exhibit some degree of constitutive internalization (Bhattacharya et al., 2004; Dale et al., 2001a,b; Fourgeaud et al., 2003; Pula et al., 2004), and undergo rapid agonist-induced internalization (Dale et al., 2001a,b; Doherty et al., 1999; Mundell et al., 2001, 2003; Sallese et al., 2000). Also consistent with our observations, live imaging in HEK cells revealed minimal recovery of surface GFP-mGluR1a following internalization during continued presence of agonist or within $10 \mathrm{~min}$ of agonist washout (Doherty et al., 1999). These studies primarily focused on classical questions related to GPCR internalization such as involvement of GPCR kinases (GRKs) and arrestins 
along with the cellular mechanisms of endocytosis such as clathrin- and dynamin-dependence (Dhami and Ferguson, 2006). While similar examination for mGluR7 trafficking requires future investigation a recent report revealed that group III mGluR activation with L-AP4 in dopaminergic neurons activates the MAP kinase pathway through a mechanism requiring $\beta$-arrestin2, dynamin, and src (Jiang et al., 2006). As such GPCR mediated MAP kinase activation proceeds following arrestin recruitment and subsequent clathrin/dynamin dependent endocytosis (reviewed in Lefkowitz and Shenoy, 2005), these findings are consistent with agonist-induced group III mGluR internalization via a classical arrestin-, dynamin-, and clathrindependent pathway. Interestingly, activation of the MAP kinase pathway in dopaminergic neurons by L-AP4 provided neuroprotection against rotenone toxicity (Jiang et al., 2006). Similar neuroprotective effects of group III mGluR activation have been observed in diverse models of neurotoxicity (Flor et al., 2002). One impediment to such research has been the inability to directly assess a potential role for mGluR7 since the only available agonist (L-AP4) could not distinguish between group III mGluRs. Our present findings now indicate that AMN082 should allow selective examination of the roles for mGluR7 in transmission and neuroprotection regardless of whether receptor activation, internalization, or both are required.

\section{Acknowledgements}

The authors thank Vincent Schram, and Louis Dye for expert technical assistance with pHluorin-mGluR7 imaging performed in the NICHD microscopy and imaging core (MIC). We additionally thank Susan Smith for preparing hippocampal cultures and Carolyn Smith for providing assistance with confocal imaging in the NINDS imaging facility. Myc-mGluR7 constructs were kindly provided by Drs. L. Fagni and Federica Bertaso. Superecliptic pHluorin was a generous gift from Drs. Jeremy Henley and Mike Ashby. Drs. Jinny Kim and Dax Hoffman provided the modified Sindbis viral vector SINrep(nsP2S726) and helper vector DH-BB (tRNA/TE12). This work was supported by an NICHD intramural award to C.J.M with additional support by an NINDS intramural award to K.W.R. K.A.P. is an NIH Visiting Fellow and a CIHR Fellow.

\section{References}

Alexander, G.M., Godwin, D.W., 2005. Presynaptic inhibition of corticothalamic feedback by metabotropic glutamate receptors. J. Neurophysiol. 94, $163-175$

Ashby, M.C., De La Rue, S.A., Ralph, G.S., Uney, J., Collingridge, G.L., Henley, J.M., 2004. Removal of AMPA receptors (AMPARs) from synapses is preceded by transient endocytosis of extrasynaptic AMPARs. J. Neurosci. 24, 5172-5176.

Bhattacharya, M., Babwah, A.V., Godin, C., Anborgh, P.H., Dale, L.B., Poulter, M.O., Ferguson, S.S., 2004. Ral and phospholipase D2-dependent pathway for constitutive metabotropic glutamate receptor endocytosis. J. Neurosci. 24, 8752-8761.
Billups, B., Graham, B.P., Wong, A.Y., Forsythe, I.D., 2005. Unmasking group III metabotropic glutamate autoreceptor function at excitatory synapses in the rat CNS. J. Physiol. 565, 885-896.

Bouschet, T., Martin, S., Henley, J.M., 2005. Receptor-activity-modifying proteins are required for forward trafficking of the calcium-sensing receptor to the plasma membrane. J. Cell Sci. 118, 4709-4720.

Claing, A., Laporte, S.A., Caron, M.G., Lefkowitz, R.J., 2002. Endocytosis of $\mathrm{G}$ protein-coupled receptors: roles of $\mathrm{G}$ protein-coupled receptor kinases and beta-arrestin proteins. Prog. Neurobiol. 66, 61-79.

Conn, P.J., 2003. Physiological roles and therapeutic potential of metabotropic glutamate receptors. Ann. N.Y. Acad. Sci. 1003, 12-21.

Conn, P.J., Niswender, C.M., 2006. mGluR7's lucky number. Proc. Natl. Acad. Sci. USA 103, 251-252.

Conn, P.J., Pin, J.P., 1997. Pharmacology and functions of metabotropic glutamate receptors. Annu. Rev. Pharmacol. Toxicol. 37, 205-237.

Dale, L.B., Babwah, A.V., Bhattacharya, M., Kelvin, D.J., Ferguson, S.S., 2001a. Spatial-temporal patterning of metabotropic glutamate receptormediated inositol 1,4,5-triphosphate, calcium, and protein kinase $\mathrm{C}$ oscillations: protein kinase $\mathrm{C}$-dependent receptor phosphorylation is not required. J. Biol. Chem. 276, 35900-35908.

Dale, L.B., Bhattacharya, M., Seachrist, J.L., Anborgh, P.H., Ferguson, S.S., 2001b. Agonist-stimulated and tonic internalization of metabotropic glutamate receptor 1a in human embryonic kidney 293 cells: agonist-stimulated endocytosis is beta-arrestin1 isoform-specific. Mol. Pharmacol. 60, 12431253.

Demaurex, N., 2002. pH Homeostasis of cellular organelles. News Physiol. Sci. 17, 1-5.

Dev, K.K., Nakanishi, S., Henley, J.M., 2001. Regulation of mGlu(7) receptors by proteins that interact with the intracellular C-terminus. Trends Pharmacol. Sci. 7, 355-361.

Dhami, G.K., Ferguson, S.S., 2006. Regulation of metabotropic glutamate receptor signaling, desensitization and endocytosis. Pharmacol. Ther. 111, 260-271.

Doherty, A.J., Coutinho, V., Collingridge, G.L., Henley, J.M., 1999. Rapid internalization and surface expression of a functional, fluorescently tagged G-protein-coupled glutamate receptor. Biochem. J. 341 (Pt. 2), 415-422.

Ferguson, S.S., 2001. Evolving concepts in G protein-coupled receptor endocytosis: the role in receptor desensitization and signaling. Pharmacol. Rev. $53,1-24$.

Flor, P.J., Battaglia, G., Nicoletti, F., Gasparini, F., Bruno, G., 2002. Neuroprotective activity of metabotropic glutamate receptor ligands. Adv. Exp. Med. Biol. 513, 197-223.

Fourgeaud, L., Bessis, A.S., Rossignol, F., Pin, J.P., Olivo-Marin, J.C., Hemar, A., 2003. The metabotropic glutamate receptor mGluR5 is endocytosed by a clathrin-independent pathway. J. Biol. Chem. 278, 12222-12230.

Iacovelli, L., Bruno, V., Salvatore, L., Melchiorri, D., Gradini, R., Caricasole, A., Barletta, E., De Blasi, A., Nicoletti, F., 2002. Native group-III metabotropic glutamate receptors are coupled to the mitogenactivated protein kinase/phosphatidylinositol-3-kinase pathways. J. Neurochem 82, 216-223.

Iacovelli, L., Capobianco, L., Iula, M., Di Giorgi Gerevini, V., Picascia, A., Blahos, J., Melchiorri, D., Nicoletti, F., De Blasi, A., 2004. Regulation of mGlu4 metabotropic glutamate receptor signaling by type-2 G-protein coupled receptor kinase (GRK2). Mol. Pharmacol. 65, 1103-1110.

Jacob, T.C., Bogdanov, Y.D., Magnus, C., Saliba, R.S., Kittler, J.T., Haydon, P.G., Moss, S.J., 2005. Gephyrin regulates the cell surface dynamics of synaptic GABAA receptors. J. Neurosci. 25, 10469-10478.

Jiang, Q., Yan, Z., Feng, J., 2006. Activation of group III metabotropic glutamate receptors attenuates rotenone toxicity on dopaminergic neurons through a microtubule-dependent mechanism. J. Neurosci. 26, 4318-4328.

Kim, J., Dittgen, T., Nimmerjahn, A., Waters, J., Pawlak, V., Helmchen, F., Schlesinger, S., Seeburg, P.H., Osten, P., 2004. Sindbis vector SINrep(nsP2S726): a tool for rapid heterologous expression with attenuated cytotoxicity in neurons. J. Neurosci. Methods 133, 81-90.

Klaasse, E.C., van den Hout, G., Roerink, S.F., de Grip, W.J., Ijzerman, A.P., Beukers, M.W., 2005. Allosteric modulators affect the internalization of human adenosine A1 receptors. Eur. J. Pharmacol. 522, 1-8. 
Kopec, C.D., Li, B., Wei, W., Boehm, J., Malinow, R., 2006. Glutamate receptor exocytosis and spine enlargement during chemically induced long-term potentiation. J. Neurosci. 26, 2000-2009.

Lavezzari, G., McCallum, J., Dewey, C.M., Roche, K.W., 2004. Subunit-specific regulation of NMDA receptor endocytosis. J. Neurosci. 24, 6383-6391.

Lefkowitz, R.J., Shenoy, S.K., 2005. Transduction of receptor signals by betaarrestins. Science 308, 512-517.

Macek, T.A., Schaffhauser, H., Conn, P.J., 1998. Protein kinase C and A3 adenosine receptor activation inhibit presynaptic metabotropic glutamate receptor (mGluR) function and uncouple mGluRs from GTP-binding proteins. J. Neurosci. 18, 6138-6146.

Mathiesen, J.M., Ramirez, M.T., 2006. The metabotropic glutamate receptor 4 is internalized and desensitized upon protein kinase $\mathrm{C}$ activation. $\mathrm{Br}$. J. Pharmacol.

May, L.T., Lin, Y., Sexton, P.M., Christopoulos, A., 2005. Regulation of M2 muscarinic acetylcholine receptor expression and signaling by prolonged exposure to allosteric modulators. J. Pharmacol. Exp. Ther. 312, 382-390.

Miesenbock, G., De Angelis, D.A., Rothman, J.E., 1998. Visualizing secretion and synaptic transmission with $\mathrm{pH}$-sensitive green fluorescent proteins. Nature 394, 192-195.

Mitsukawa, K., Yamamoto, R., Ofner, S., Nozulak, J., Pescott, O., Lukic, S., Stoehr, N., Mombereau, C., Kuhn, R., McAllister, K.H., van der Putten, H., Cryan, J.F., Flor, P.J., 2005. A selective metabotropic glutamate receptor 7 agonist: activation of receptor signaling via an allosteric site modulates stress parameters in vivo. Proc. Natl. Acad. Sci. USA 102, 18712-18717.

Mundell, S.J., Matharu, A.L., Pula, G., Roberts, P.J., Kelly, E., 2001. Agonistinduced internalization of the metabotropic glutamate receptor $1 \mathrm{a}$ is arrestin- and dynamin-dependent. J. Neurochem. 78, 546-551.

Mundell, S.J., Pula, G., Carswell, K., Roberts, P.J., Kelly, E., 2003. Agonistinduced internalization of metabotropic glutamate receptor 1A: structural determinants for protein kinase $\mathrm{C}$ - and $\mathrm{G}$ protein-coupled receptor kinase-mediated internalization. J. Neurochem. 84, 294-304.

Niswender, C.M., Jones, C.K., Conn, P.J., 2005. New therapeutic frontiers for metabotropic glutamate receptors. Curr. Top. Med. Chem. 5, 847-857.

Pelkey, K.A., Lavezzari, G., Racca, C., Roche, K.W., McBain, C.J., 2005. mGluR7 is a metaplastic switch controlling bidirectional plasticity of feed forward inhibition. Neuron 46, 89-102.

Perroy, J., Prezeau, L., De Waard, M., Shigemoto, R., Bockaert, J., Fagni, L., 2000. Selective blockade of P/Q-type calcium channels by the metabotropic glutamate receptor type 7 involves a phospholipase $\mathrm{C}$ pathway in neurons. J. Neurosci. 20, 7896-7904.

Pin, J.P., Acher, F., 2002. The metabotropic glutamate receptors: structure, activation mechanism and pharmacology. Curr. Drug Targets CNS Neurol Disord. 1, 297-317.
Pula, G., Mundell, S.J., Roberts, P.J., Kelly, E., 2004. Agonist-independent internalization of metabotropic glutamate receptor $1 \mathrm{a}$ is arrestin- and clathrin-dependent and is suppressed by receptor inverse agonists. J. Neurochem. 89, 1009-1020.

Sachpatzidis, A., Benton, B.K., Manfredi, J.P., Wang, H., Hamilton, A., Dohlman, H.G., Lolis, E., 2003. Identification of allosteric peptide agonists of CXCR4. J. Biol. Chem. 278, 896-907.

Sallese, M., Salvatore, L., D’Urbano, E., Sala, G., Storto, M., Launey, T., Nicoletti, F., Knopfel, T., De Blasi, A., 2000. The G-protein-coupled receptor kinase GRK4 mediates homologous desensitization of metabotropic glutamate receptor 1. Faseb J. 14, 2569-2580.

Sankaranarayanan, S., Ryan, T.A., 2000. Real-time measurements of vesicleSNARE recycling in synapses of the central nervous system. Nat. Cell Biol. 2, 197-204.

Schoepp, D.D., 2001. Unveiling the functions of presynaptic metabotropic glutamate receptors in the central nervous system. J. Pharmacol. Exp. Ther. $299,12-20$.

Schoppa, N.E., Westbrook, G.L., 1997. Modulation of mEPSCs in olfactory bulb mitral cells by metabotropic glutamate receptors. J. Neurophysiol. $78,1468-1475$.

Shen, W., Slaughter, M.M., 1998. Metabotropic and ionotropic glutamate receptors regulate calcium channel currents in salamander retinal ganglion cells. J. Physiol. 510 (Pt. 3), 815-828.

Shipe, W.D., Wolkenberg, S.E., Williams Jr., D.L., Lindsley, C.W., 2005. Recent advances in positive allosteric modulators of metabotropic glutamate receptors. Curr. Opin. Drug Discov. Dev. 8, 449-457.

Sternweis, P.C., Robishaw, J.D., 1984. Isolation of two proteins with high affinity for guanine nucleotides from membranes of bovine brain. J. Biol. Chem. 259, 13806-13813.

Swartz, K.J., Merritt, A., Bean, B.P., Lovinger, D.M., 1993. Protein kinase C modulates glutamate receptor inhibition of $\mathrm{Ca} 2+$ channels and synaptic transmission. Nature 361, 165-168.

Tong, Q., Kirchgessner, A.L., 2003. Localization and function of metabotropic glutamate receptor 8 in the enteric nervous system. Am. J. Physiol. Gastrointest. Liver Physiol. 285, G992-G1003.

Toulme, E., Soto, F., Garret, M., Boue-Grabot, E., 2006. Functional properties of internalization-deficient $\mathrm{P} 2 \mathrm{X} 4$ receptors reveal a novel mechanism of ligandgated channel facilitation by ivermectin. Mol. Pharmacol. 69, 576-587.

Tzounopoulos, T., Janz, R., Sudhof, T.C., Nicoll, R.A., Malenka, R.C., 1998. A role for cAMP in long-term depression at hippocampal mossy fiber synapses. Neuron 21, 837-845.

Yudowski, G.A., Puthenveedu, M.A., von Zastrow, M., 2006. Distinct modes of regulated receptor insertion to the somatodendritic plasma membrane. Nat. Neurosci. 9, 622-627. 